\title{
Design concept of the magnetic horn system for the T2K neutrino beam
}

\author{
$\operatorname{AUTHOR}(\mathrm{S}):$ \\ Ichikawa, A.K.
}

\section{CITATION:}

Ichikawa, A.K.. Design concept of the magnetic horn system for the T2K neutrino beam. Nuclear Instruments and Methods in Physics Research Section A: Accelerators,

Spectrometers, Detectors and Associated Equipment 2012, 690: 27-33

ISSUE DATE:

2012-10-21

URL:

http://hdl.handle.net/2433/160124

\section{RIGHT:}

C 2012 Elsevier B.V.; この論文は出版社版でありません。引用の際には 出版社版をご確認ご利用ください。; This is not the published version. Please cite only the published version. 


\title{
Design concept of the magnetic horn system for the T2K neutrino beam
}

\author{
A.K.Ichikawa ${ }^{\mathrm{a}}$ \\ ${ }^{a}$ Department of Physics, Kyoto University, Kyoto 606-8502, Japan
}

\begin{abstract}
The design concept of the magnetic horn system for the T2K neutrino oscillation experiment is described. We proposed a three-horn system with optics similar to a conventional two-horn system, aiming to focus relatively low-momentum pions under a high beam intensity environment. Good focusing efficiency was obtained with compact horns and a rather large inner conductor radius for the first horn. The geometry of the decay volume was also optimized. The intensity and quality of the obtained neutrino flux is satisfactory to achieve the T2K physics goals.
\end{abstract}

Keywords: magnetic horn, neutrino beam, T2K

\section{Introduction}

To explore the still not-understood features of neutrinos, experiments using muon neutrino beams are actively being conducted or planned. One of the most important aspects of these experiments is the intensity of the beam, because neutrino interacts extremely rarely and may travel hundreds of kilometers before detection. In a so called conventional beam, muon neutrinos are produced as decay products of pions. The pions are produced via the interaction of accelerated protons with a target material. To increase the intensity of neutrinos in a particular direction, pions are focused forward by the electromagnetic horns proposed by van der Meer[1]. Horns are essentially aluminum coaxial conductors: an inner conductor and an outer conductor. A toroidal magnetic field is generated between the conductors when electrical current flows through the inner conductor and returns through the outer conductor. Usually, pulsed currents are applied synchronously with the proton beam pulse. 
A conventional neutrino beam is called a "super beam" when the proton beam power is a order of megawatts. In this case, equipment to generate the neutrino beam, such as target and horns, has to withstand both radiation damage and instantaneous thermal stress and have a cooling system for the heat load from radiation.

In this paper, we describe the design concept of the horn system for the T2K experiment[2][3]. T2K is a long-baseline neutrino oscillation experiment, in which neutrinos are generated by the J-PARC accelerator complex at Tokai-mura, Japan and are detected by a water Cherenkov detector (SuperKamiokande, Super-K) after traveling $295 \mathrm{~km}$. A $30 \mathrm{GeV}$ proton beam is extracted from the J-PARC accelerator every 2 to 3 seconds. Each pulse, or so-called spill, contains 8 bunches in about $5 \mu \mathrm{s}$. The design intensity of the proton beam is $0.75 \mathrm{MW}$ corresponding to $3.3 \times 10^{14}$ protons or $1.6 \mathrm{GJ}$ of energy per spill. T2K adopted the off-axis method[4] to generate a narrow-band neutrino beam. In this method, the neutrino beam is intentionally directed at an angle with respect to the baseline connecting the proton target and the far detector. In the T2K experiment, the off-axis angle is set at $2.5^{\circ}$ and the peak energy is $0.6 \mathrm{GeV}$. With this narrowly tuned beam, the oscillation probability at the baseline length of $295 \mathrm{~km}$ is maximized and backgrounds from high-energy neutrinos are suppressed. The momentum region of parent pions to be focused is 1 to $2 \mathrm{GeV}$, which is relatively low compared to those in the other neutrino beam facilities for long-baseline experiments such as NuMI[5] and CNGS[6]. These low-momentum pions are emitted from the target at relatively large angles (around $100 \mathrm{mrad}$ ). Hence the design of the target and horn is optimized to focus these low momentum and large emission angle pions.

Secs. 2 and 3 respectively describe the conceptual design procedure of the target and horns. Section 4 describes the optimization of the decay volume direction and shape. Section 5 shows the expected neutrino flux from this conceptual design.

\section{Target}

We chose graphite as a hadron production target. Materials heavier than graphite cannot withstand the heat load from radiation. The target length is 1.9 interaction lengths ( $85 \%$ of protons interact ), i.e. $90 \mathrm{~cm}$ for $\rho=1.8$ $\mathrm{g} / \mathrm{cm}^{3}$. 


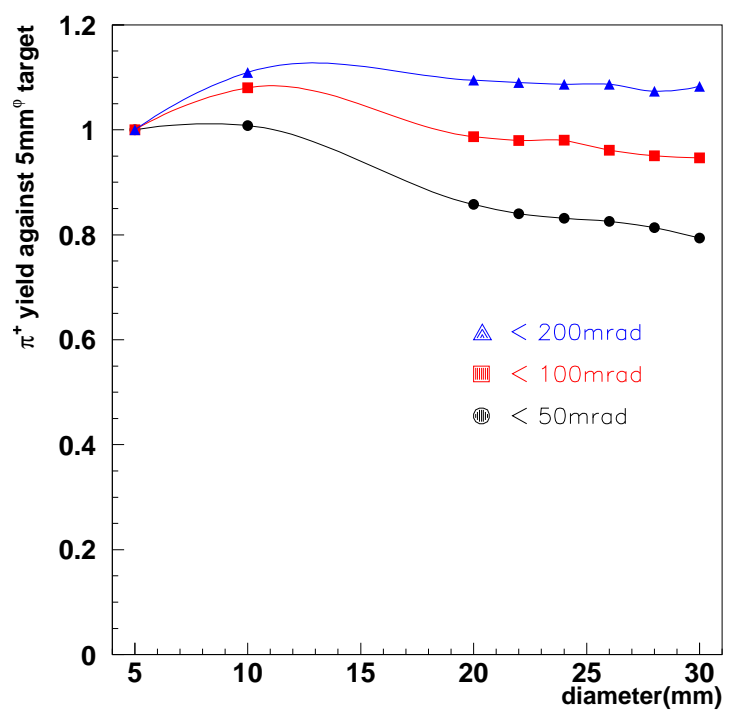

Figure 1: Dependence of $\pi^{+}$yield on target diameter for each region of $\pi^{+}$emission angle. The relative yield against that with a $5 \mathrm{~mm}$ diameter target is shown. The primary proton beam is assumed to have a Gaussian distribution and its size is adjusted so that $99 \%$ of the protons hit the target.

Figure 1 shows the dependence of the $\pi^{+}$yield on the diameter of the target, calculated by the GEANT3 simulation package[9] with the GCALOR hadron production model[10]. In the region from $20 \mathrm{~mm}$ to $30 \mathrm{~mm}$ in diameter, the effect of pion absorption due to the target material is not so large. On the other hand, heat load from radiation limits the lower bound for the target diameter to about $26 \mathrm{~mm}$. Therefore, the diameter of $26 \mathrm{~mm}$ was chosen[7]. Then, in the following study, the primary proton beam is assumed to have a 2-dimensional Gaussian distribution whose r.m.s. is 4.2 $\mathrm{mm}$ in each projection so that $99 \%$ of the protons hit the target.

\section{Horn}

The typical transverse momentum of pions to be focused in the T2K experiment is $0.4 \mathrm{GeV} / \mathrm{c}$. To focus such pions, a field integral of $1.3 \mathrm{Tm}$ is necessary. Figure 2 shows trajectories of particles having typical momenta $(1.0 \mathrm{GeV} / c \sim 4.0 \mathrm{GeV} / c)$ and typical angle in the horn magnetic field when the production target is placed just upstream of the horn. As can be seen 


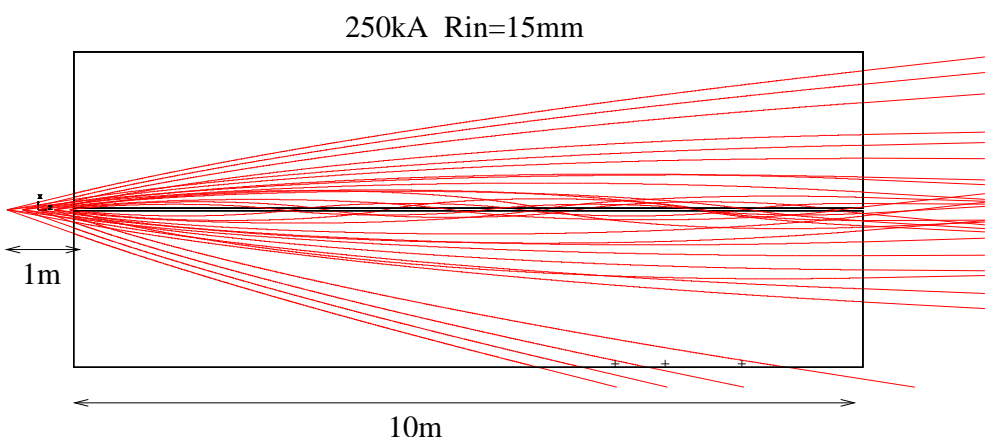

Figure 2: Trajectories of particles having typical momenta $(1.0 \mathrm{GeV} / c \sim 4.0 \mathrm{GeV} / c)$ and typical angles in a horn magnetic field when the target is placed upstream of the horn. Here, the horn current is $250 \mathrm{kA}$ and the radius of the inner conductor is $15 \mathrm{~mm}$.

from the figure, tracks are not focused yet even with a $10 \mathrm{~m}$ long horn. This is because the emission angle of pions is relatively large $(O(100) \mathrm{mrad})$ in the T2K experiment, and tracks are far away from the center axis when particles reach the horn. Since the magnetic field strength is inversely proportional to radial position, the field is too weak for those particles. Therefore, we conclude that the production target must be set inside the inner-conductor of the first horn for the T2K neutrino beam production.

From the restriction on the target diameter and cooling needs of the target[7], the outer radius of the inner-conductor is set to $26 \mathrm{~mm}$ as a first baseline design. This value is rather large compared to horns in other facilities. Then, in order to achieve required field integral, length or current has to be larger. To restrict both the length and current value in the feasible region, we adopted a three-horns scheme. In many neutrino facilities, the configuration with the two horns proposed by R. Palmer[8] has been adopted. In this setup, the inner conductor of the first horn consists of three components as shown in Fig. 3. The upstream straight tube part acts as a collector. The downstream conical part acts as a focusing lens. The middle part adds field integral for large angle pions. The shape of the downstream conical part is essential to focus pions. In our proposed scheme, the upstream part and down stream part is separated into two independent horns. Figure 4 shows a schematic view of the proposed horn system. The chosen electric current is $320 \mathrm{kA}$. The other reason to adopt the three-horn scheme is very high heat load from radiation to the first horn. A shorter first horn is preferable for cooling. Joule heating is also small with a shorter first horn. 


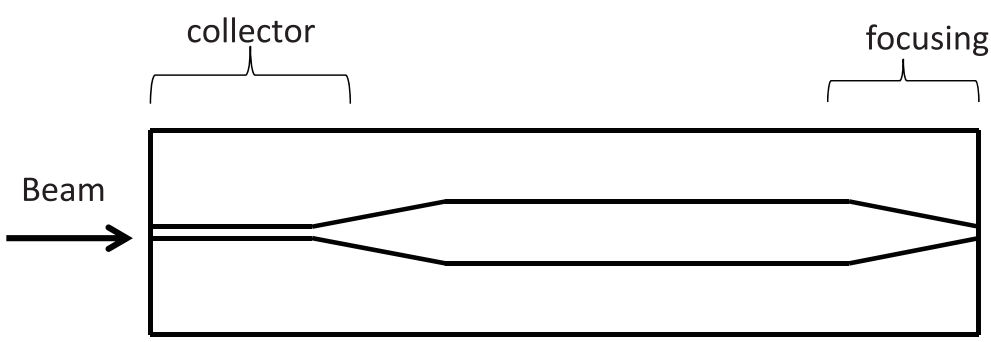

Figure 3: Conceptual shape of the first horn in the two horns system proposed by R. Palmer.

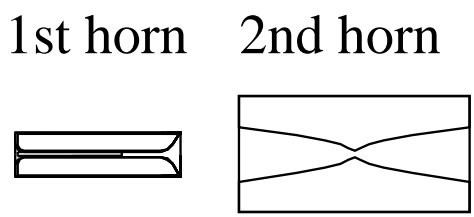

\section{$100 \mathrm{~cm}$}

\section{3rd horn}

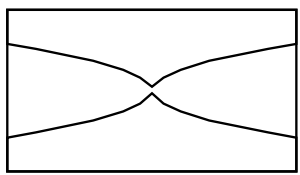

Figure 4: Schematic view of the proposed horn system.

The shapes of the inner conductors for the second and third horns were optimized to focus pions. For the purpose of both the optimization of the horn and resultant neutrino flux calculation, the GEANT3 simulation package[9] was used. The pion production inside the target was simulated by the GCALOR model[10], which is built in to GEANT3. Particles are traced inside GEANT3 in both the magnetic field and the field-free region.

The optimization was done by two steps: a general optimization by a simple method and then fine tuning.

\section{1. general first step optimization}

For the general optimization of the second and third horns, the necessary field length is calculated for each pion exiting the upstream horn in the following manner. The track is extrapolated linearly to the most downstream plane of the horn being optimized. The field strength there is calculated. The necessary field length to focus that pion parallel to the horn axis is calculated from the field strength and pion angle from the horn axis, assuming that the field strength is constant along the track. The ideal incident positions (radial and $z$ ) are calculated taking $z$ as the horn downstream end minus the necessary field length and are plotted for each track in Fig.5 in case of the 


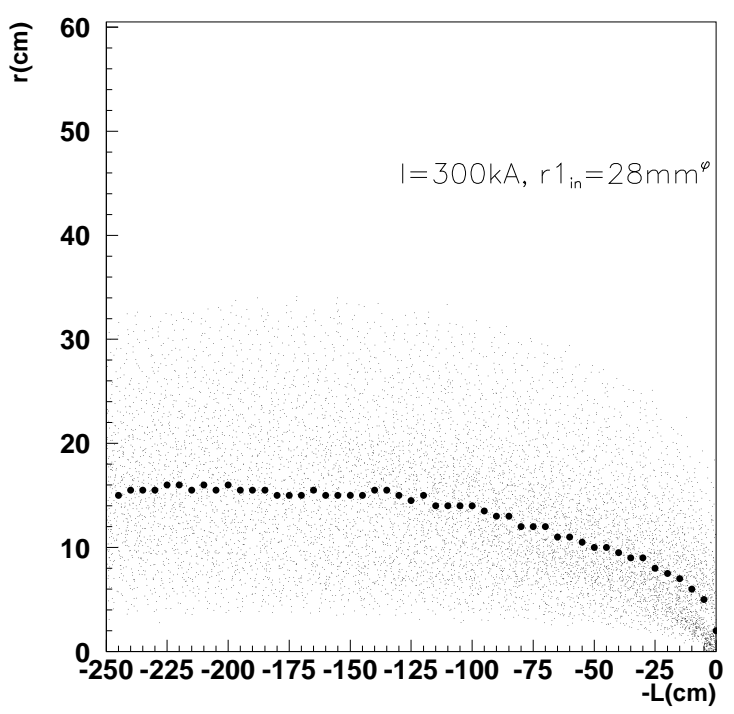

Figure 5: Ideal incident position $(r, z)$ on the second horn for each track from the first horn. $z=0$ corresponds to the downstream end of the horn. The circles are medians of $\mathrm{r}$ for each z bins.

second horn. Here $z$ is the position along the horn axis. The shape of the inner conductor was determined by taking the median of the $\mathrm{r}$ distribution for each $\mathrm{z}$.

\subsection{Fine tuning}

Neutrino yields were calculated with various set-ups for further fine tuning starting from the one obtained in the general first step optimization. In the first optimization step, we obtained an inner conductor shape close to a parabola for the second and third horns. For the fine tuning, the shape of the inner conductor of the second and third horns is changed to two symmetric paraboloids with a waist at the center. This is because we found that higher neutrino yield is obtained with two symmetric paraboloids. It is also superior in technical points: it is easy to construct and strong against the Lorentz force. The parameters tuned are,

- electrical current:I

- (outer radius of) inner-conductor: $r_{i n}$ 


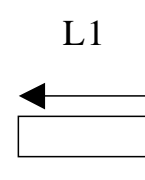

Horn 1
D2

L2

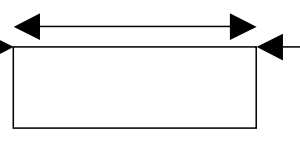

Horn2
D3

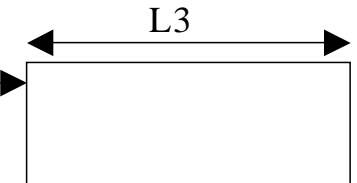

Horn3

Figure 6: Definition of some of the tuning parameters.

- first horn length:L1

- distance between the first horn and second horn:D2

- shape parameter for the second horn inner-conductor:a2

- shape parameter for the third horn inner-conductor:a3

The $a 2$ and $a 3$ are the coefficients of the parabola. The following parameters are fixed:

- second horn length: $L 2=2 \mathrm{~m}$

- third horn length: $L 3=2.5 \mathrm{~m}$

- total length (L1+D2+L2+D3+L3):11.5 m

Figure 6 shows the definitions of some of the geometrical parameters. The obtained relative neutrino yields for various set-ups are plotted in Fig.7. The off-axis angle was fixed to 2.5 degrees. At around $I=320 \mathrm{kA}$, a slightly better neutrino yield is obtained with $r_{i n}=28 \mathrm{~mm}$ rather than $26 \mathrm{~mm}$. Although it is decreased at $30 \mathrm{~mm}$, we adopt $30 \mathrm{~mm}$ radius as the first horn's inner conductor radius based on the consideration on the thermal stress due to radiation heating. The flux of produced charged particles is much higher at smaller radial position. It was found that the thermal stress generated in the aluminum conductor is higher than the allowable limit for the case of 26 $\mathrm{mm}$ inner conductor radius. The difference in yield between $L 1=140 \mathrm{~cm}$ and $150 \mathrm{~cm}$ is small. Therefore, we adopt length of $140 \mathrm{~cm}$ for the first horn. The schematic view of the tuned horn system is drawn in Fig.4.

\subsection{Outer radius}

The radius of the outer conductor was determined sufficiently large to keep the muon neutrino yield(Fig.8). 


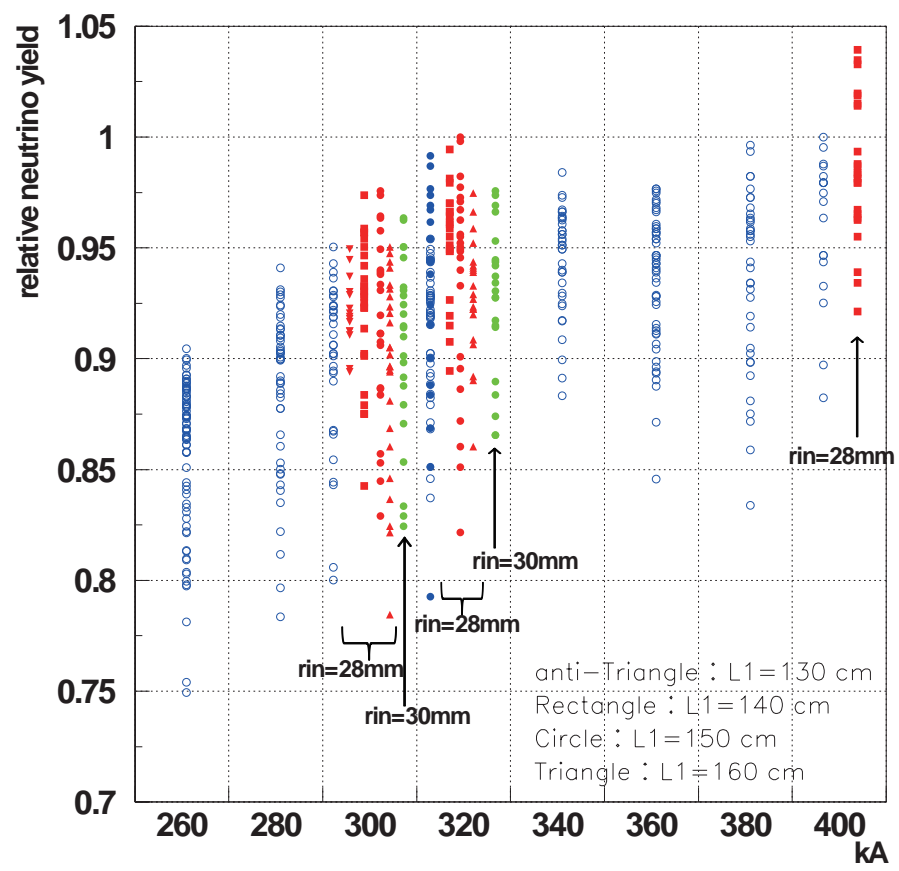

Figure 7: Relative neutrino yields for various set-ups. Each point corresponds to one setup. The radius of the first horn is $26 \mathrm{~mm}$ unless otherwise noted. Markers in each column corresponds to one set of $a 1, a 2$ and $D 2$ parameters.

\subsection{Length and Position of the Target}

With the obtained optimized horn system, the dependence of the neutrino yield on the target length and target position along the beam is examined. See Fig.9 and 10. Note that the change of the yield is less than $1 \%$ for the various position of the target in this region. Therefore it can be determined from the engineering point of view within this region.

\subsection{Comparison with perfect focusing}

In Fig. 11, the on-axis neutrino flux obtained with the optimized horn system is compared with that assuming perfect focusing. With the perfectfocusing horn, all forward-produced pions are assumed to be focused to perfectly parallel to the primary beam. Instead of the off-axis flux, the on-axis neutrino flux is used for the comparison in order to see the dependence on the pion momentum, which is roughly twice the resultant neutrino energy. The focusing efficiency at around $2 \mathrm{GeV}$ is very high even though the radius of our first horn's inner conductor is as large as $30 \mathrm{~mm}$. 

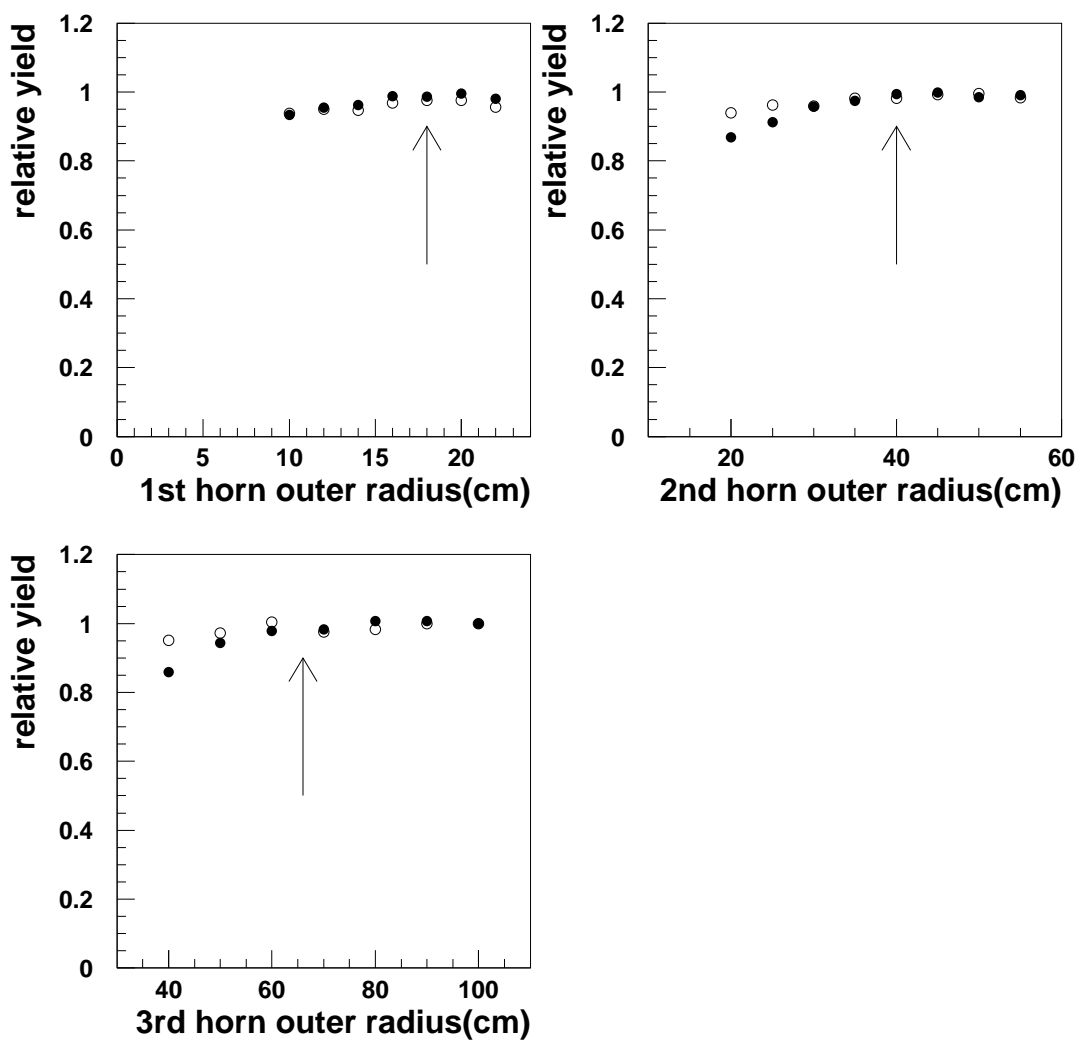

Figure 8: Relative neutrino yield as a function of horn outer-conductor radius.Black and white circles correspond to muon neutrino and electron neutrino, respectively. The arrows indicate the adopted radius.

\subsection{Pion absorption by aluminum}

So far, the optimization was done without material. The actual inner conductors of the T2K horns are made of aluminum. Its thickness must be $3 \mathrm{~mm}$ to withstand the stress from the Lorentz force. Figure 12 shows the effect of the particle absorption or interaction with the aluminum. Even with the three-horn scheme, the reduction of neutrino yield due to pion absorption by aluminum is at the same level as in a two horn-scheme:20\%. This feature is expected from the fact that the optics of our three-horn system is basically the same as the two horn system. 


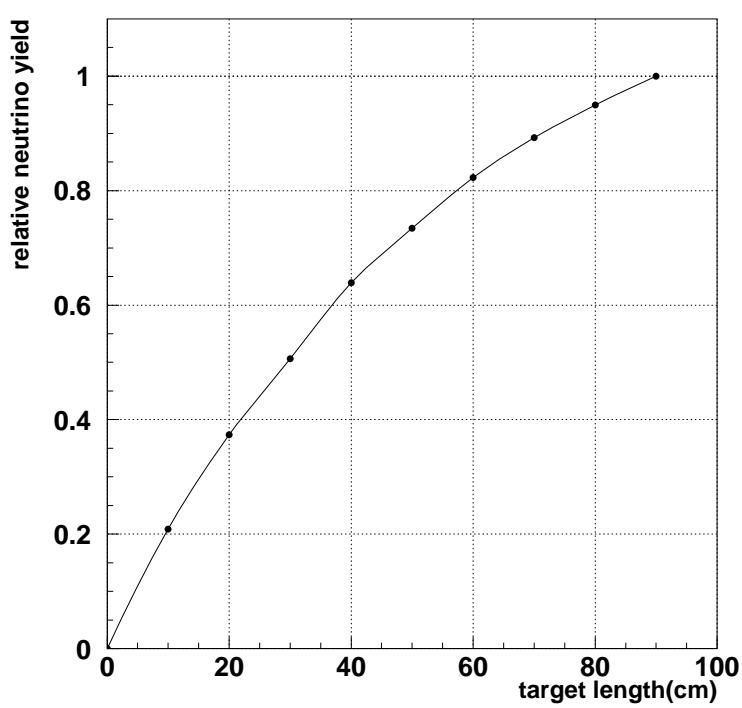

Figure 9: Relative neutrino yield as a function of the target length. The upstream position of the target is fixed to the upstream end of the first horn.

\section{Decay Volume direction and shape}

\subsection{Off-Axis beam angle}

The peak energy of the neutrino beam is tuned at the oscillation maximum by adjusting the off-axis angle in the T2K experiment. For the various values of $\Delta m^{2}$, the neutrino energies at the oscillation maximum for the baseline length of $295 \mathrm{~km}$ and the corresponding off-axis angles of the beam are summarized in Table 1 . The direction of the decay volume has to be determined to cover the proper off-axis angle. There is a future plan called Hyper-Kamiokande (Hyper-K)[11] to further pursue a neutrino oscillation research program, especially to search for $\mathrm{CP}$ violation in the lepton sector using the neutrino beam from J-PARC. The candidate site for the Hyper-K is located about $8 \mathrm{~km}$ south of Super-K. Figure 13 shows the schematic directional view of Super-K and the Hyper-K candidate site from the target at J-PARC. The T2K decay volume was designed to cover off-axis angle from 2 to 3 degree for both Super-K and the Hyper-K candidate site. 


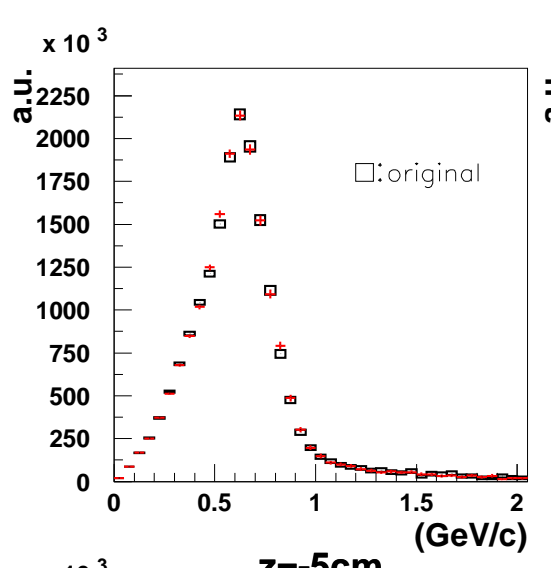

Ey $^{3}{ }_{3}$
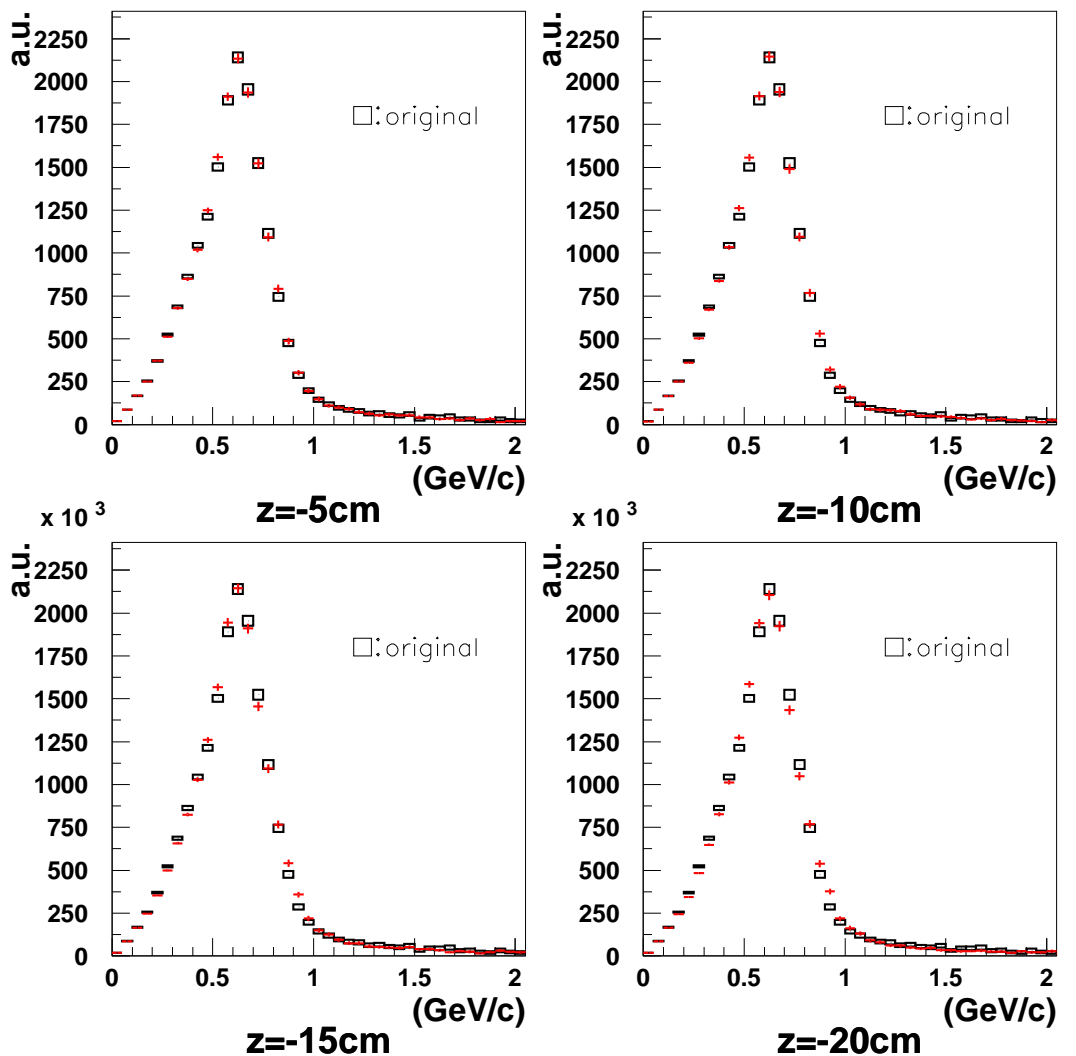

Figure 10: Comparison of the neutrino yield for various positions of the target along the beam. Here the value of $z$ corresponds to the upstream end position relative to the first horn upstream end. The target length is fixed to $90 \mathrm{~cm}$.

Table 1: $E_{\nu}$ at the oscillation maximum for a baseline length of $295 \mathrm{~km}$ for values of $\delta m_{32}^{2}$ and corresponding optimum off-axis angle.

\begin{tabular}{cccccc}
\hline \hline$\Delta m^{2}\left[10^{-3} \mathrm{eV}^{2}\right]$ & 2.04 & 2.18 & 2.75 & 3.17 & 3.28 \\
\hline$E_{\nu}[\mathrm{GeV}]$ & 0.487 & 0.520 & 0.656 & 0.756 & 0.782 \\
OA angle[deg. $]$ & 3.1 & 3.0 & 2.4 & 2.1 & 2.0 \\
\hline
\end{tabular}

\subsection{Decay Volume shape}

The distance from the target to the end of decay volume, i.e. the surface of the beam dump, is about $109 \mathrm{~m}$. Figure 14 shows the distribution of decay 


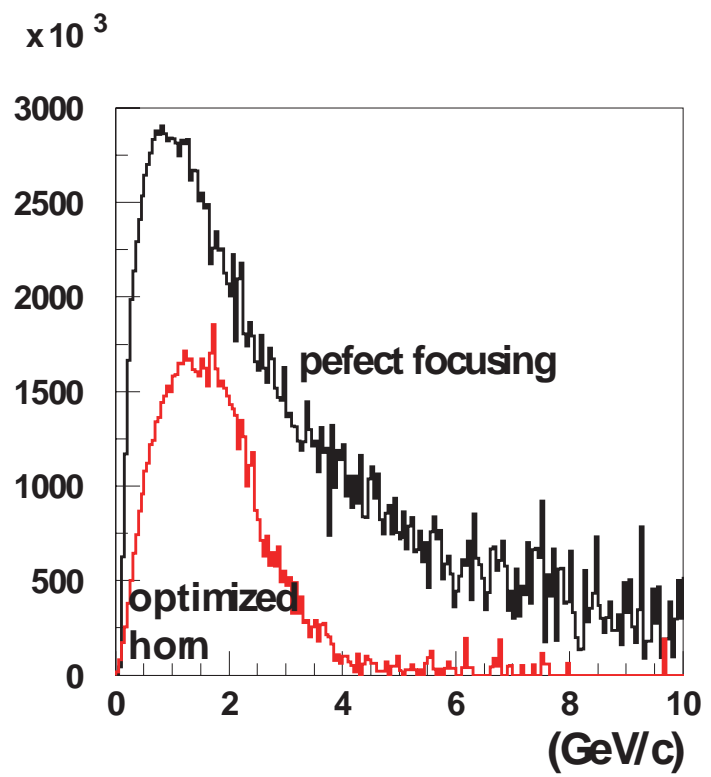

Figure 11: Comparison of on-axis muon neutrino spectra obtained with the optimized horn system and that with a perfect-focusing horn system. All forward-produced pions are assumed to be focused to perfectly parallel to the primary beam in the perfect-focusing horn system.
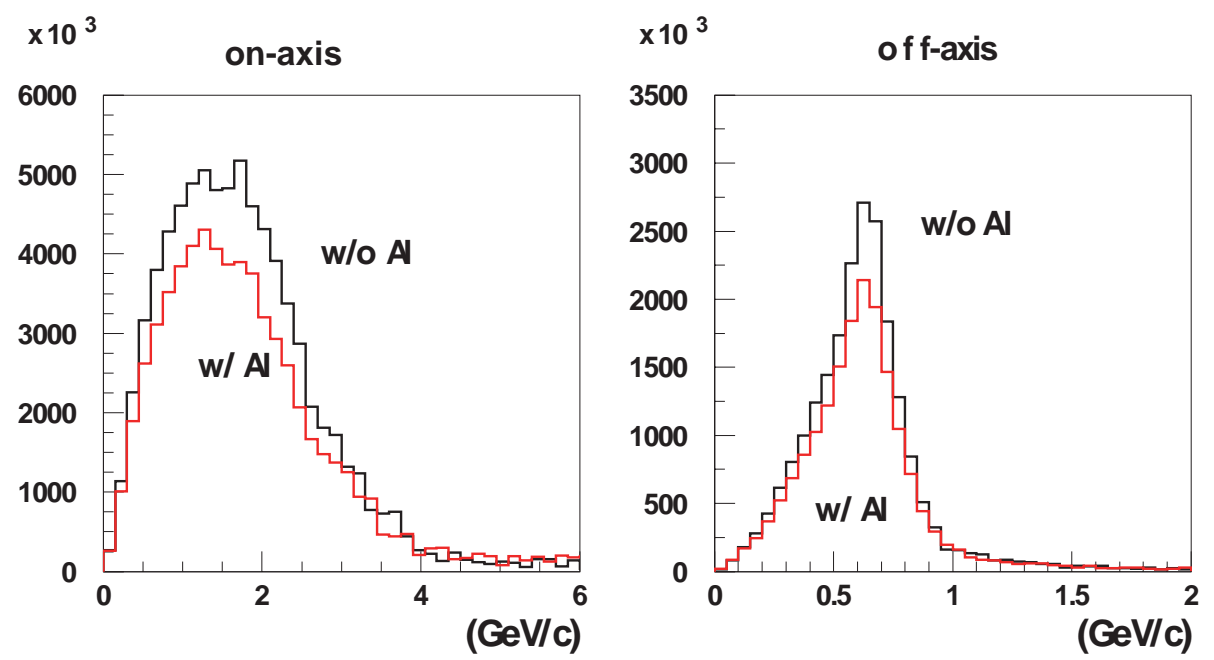

Figure 12: Muon neutrino spectra for the on-axis(left) and off-axis(right) beam with and without aluminum material. 


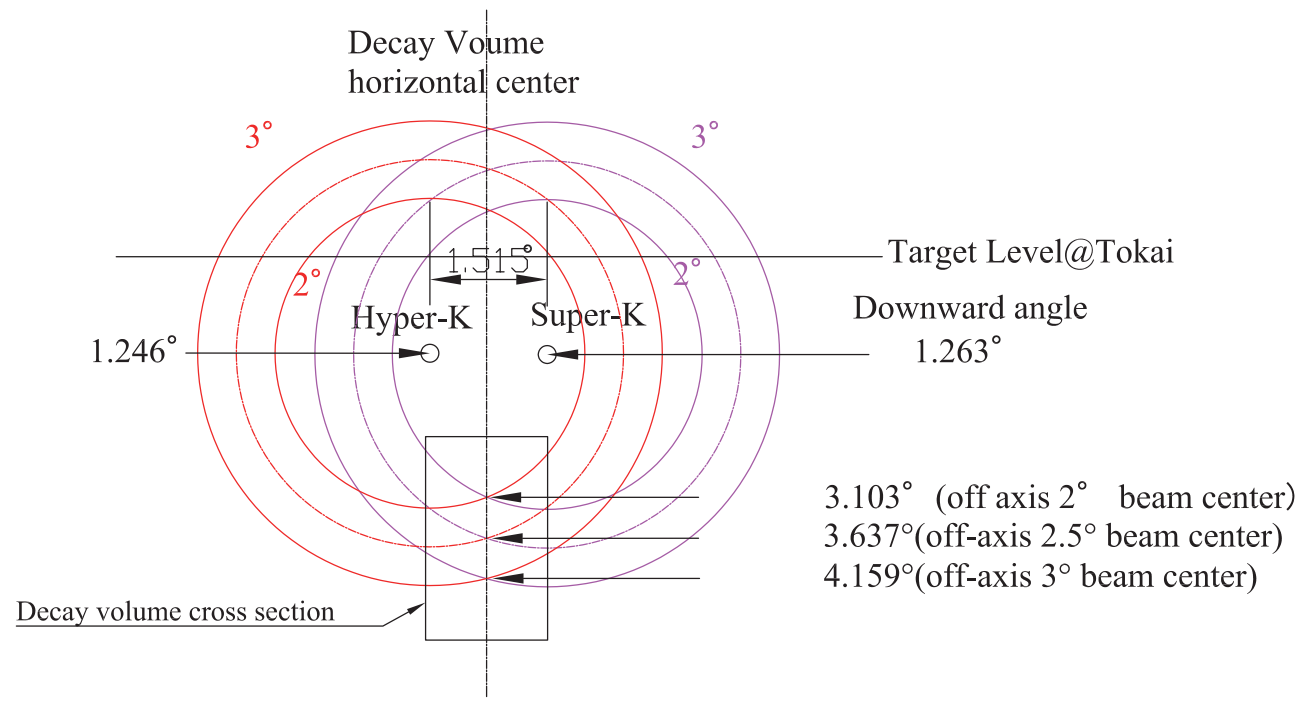

Figure 13: Schematic directional view of Super-K and Hyper-K from the neutrino beam line target at J-PARC.

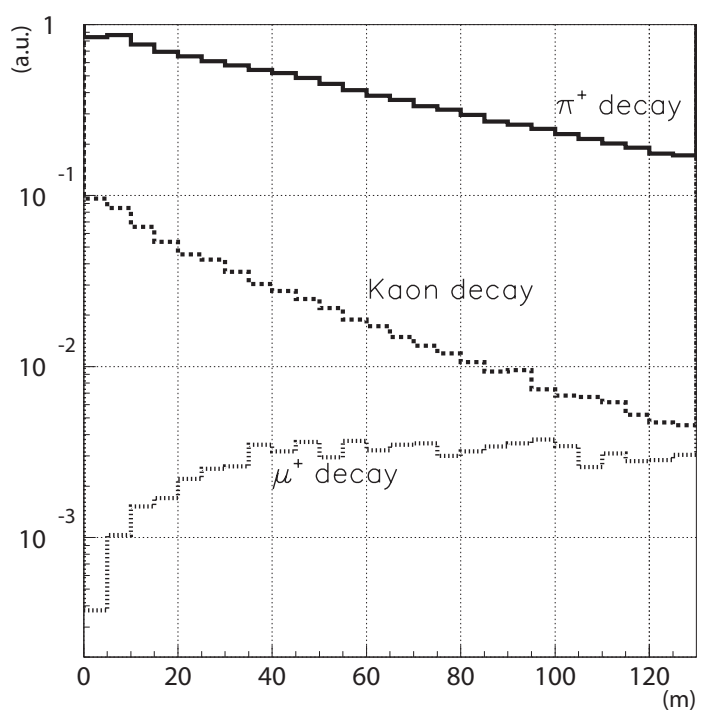

Figure 14: Distribution of decay positions of $\pi^{+}, \operatorname{kaons}\left(K^{+}\right.$and $\left.K_{L}^{0}\right)$ and $\mu^{+}$along the decay volume. Only those whose daughter neutrinos contribute to the flux at SK are plotted.

positions of $\pi^{+}, \operatorname{kaons}\left(K^{+}\right.$and $\left.K_{L}^{0}\right)$ and $\mu^{+}$along the decay volume. The cross-sectional dimension of the decay volume was determined so that the 


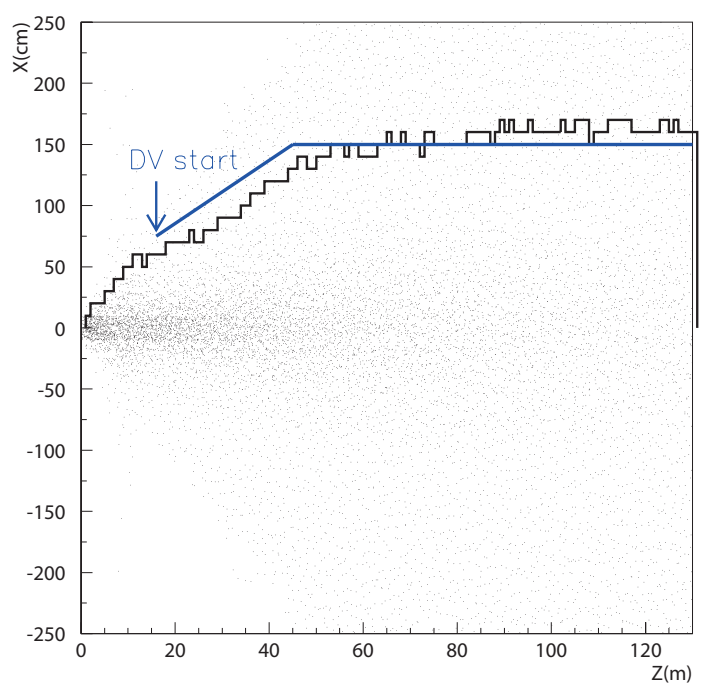

Figure 15: Position of $\pi^{+}$'s decay whose daughter neutrino has an energy around the peak of the flux. The zigzag line indicate boundary outside where the fraction of $\pi^{+}$decay is $0.04 \%$ per $1 \mathrm{~m}$ decay volume length. Two straight lines are the adopted decay volume boundary.

fractional loss of flux per unit length is constant along the volume. Figure 15 shows the positions of $\pi^{+}$decays whose daughter neutrino contributes to the peak energy of the flux. In this figure, the zigzag line indicates the boundary outside which the fraction of $\pi^{+}$decay is $0.04 \%$ per 1 meter decay volume length. Although the profile of the $\pi^{+}$flux becomes wider at the longer flight lengths, the number of surviving $\pi^{+}$decreases. Therefore at a large distance, it is not efficient to make the decay volume larger. In the actual design, the cross-sectional dimension was set at the straight lines of Fig.15. So the fractional loss due to the limited size of the decay volume is about $0.04 \%$ per 1 meter. With the 109 m-long decay volume, this number corresponds to $4.3 \%$ loss in total. The horizontal dimension was determined with these conditions. The vertical dimension was determined to accommodate varying the off-axis angle from 2 to 3 degrees. According to Fig.13, this means that the decay volume can accept a beam with a downward angle between 3.11 to 4.16 degrees. 


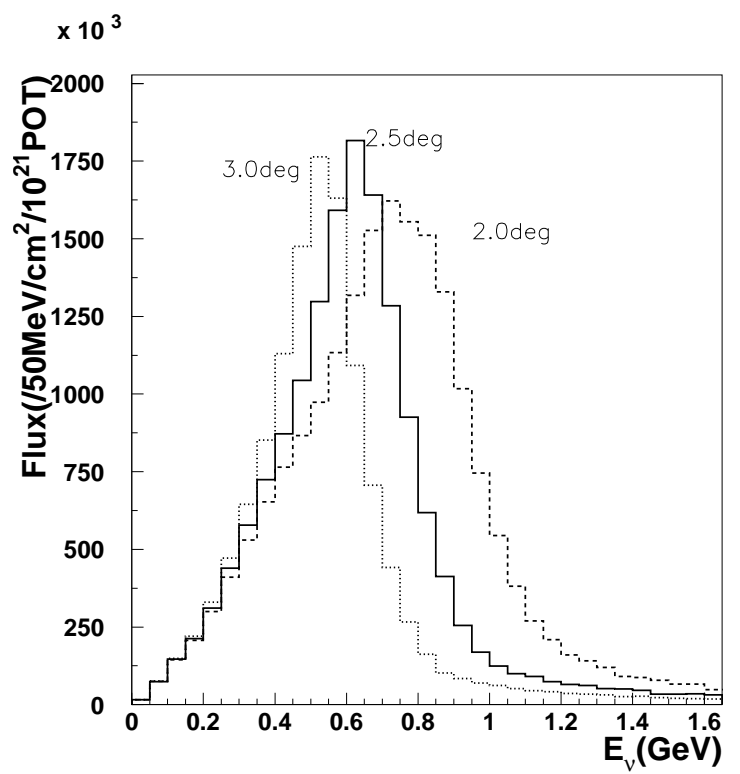

Figure 16: Expected neutrino flux at Super-K with various settings of off-axis angle. The primary proton energy is $40 \mathrm{GeV}$.

\section{Neutrino Beam}

Finally, Fig.16 shows the neutrino fluxes expected at Super-K with various settings of the off-axis angle. The primary proton energy is $40 \mathrm{GeV}$.

Figure 17 shows expected neutrino fluxes at various primary proton energies normalized to same total beam power. The spectrum shape is rather independent on the primary proton beam energy. The $30 \mathrm{GeV}$ proton beam gives about $10 \%$ higher neutrino flux than that of the $50 \mathrm{GeV}$ proton beam. The higher energy tail in the flux, which produces background to the oscillation signal, is less at $30 \mathrm{GeV}$. Therefore, if the primary proton beam power is same, the $30 \mathrm{GeV}$ beam is preferable because of its higher efficiency to produce neutrino flux and smaller high-energy tail.

\section{Summary}

The design concept of the T2K horn system is described. The key differences from the conventional existing designs arise from the required running conditions:very high beam intensity and focusing of relatively low-momentum pions. We adopted the three-horn system with essentially the same optics 


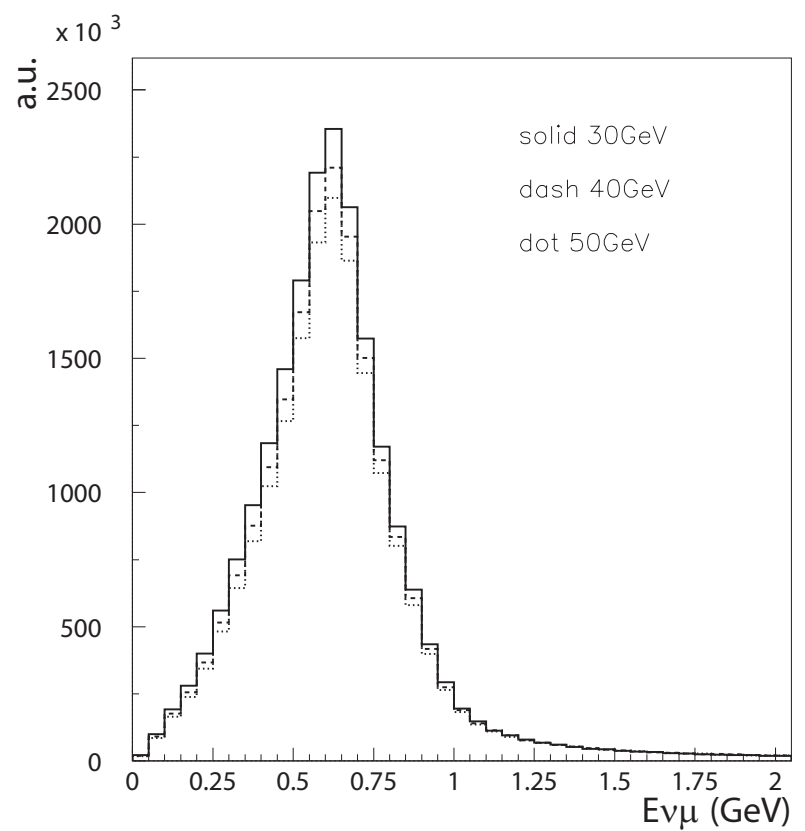

Figure 17: Neutrino flux obtained with various primary proton energies with same total beam power. The off-axis angle is 2.5 degrees.

as the conventional two horn system. After careful optimization, we keep high focusing efficiency with compact horns even though the inner conductor radius of the first horn is large compared to previous ones. The absorption of pions is comparable to that of the two-horn system. This is as expected because the optics is so similar. Together with the horn system, the geometry of the decay volume was also optimized. The intensity and quality of the obtained neutrino flux is satisfactory to enable the T2K physics goals.

\section{Acknowledgments}

The author thanks the K2K collaboration and T2K collaboration. Our $\mathrm{MC}$ simulation code is developed based from the one for the $\mathrm{K} 2 \mathrm{~K}$ collaboration. I also wish to acknowledge T. Kobayashi and Y. Yamanoi from KEK and E. D. Zimmerman from University of Colorado for valuable discussions.

\section{References}

[1] S. van der Meer, CERN-61-07, 1961. 
[2] Y.Itow et al., arXiv:hep-ex/0106019 (2001).

[3] K.Abe et al., Nuclear Instruments and Methods in Physics Research Section A 659 (2011) 106.

[4] D. Beavis, A. Carroll, I. Chiang, et al., Proposal of BNL AGS E-889 (1995).

[5] K. Anderson et al., "The NuMI Facility Technical Design Report,", fermilab-tm-design-1998-01.

[6] E. Gschwendtner et al., Proceedings of Particle Accelerator Conference (PAC 07), Albuquerque, New Mexico, 25-29 Jun 2007, pp 1601.

[7] T. Nakadaira et al., AIP Conference Proceedings 981 (2008) 290.

[8] R. B. Palmer, CERN-65-32 1965.

[9] GEANT3, A detector description and simulation tool, 1993. Application Software Group, Computing and Networks Division, CERN, Geneva.

[10] GCALOR, http://www.atlas.uni-wuppertal.de/zeitnitz/gcalor.

[11] K. Abe et al., "Letter of Intent: The Hyper-Kamiokande Experiment Detector Design and Physics Potential —," arXiv:1109.3262 [hep-ex]. 